\title{
Influence of Temperature on Phytohormone Interactions with Monolayers Obtained from Phospholipids of Wheat Calli
}

Barbara Gzyl ${ }^{\mathrm{a} *}$, Maria Filek ${ }^{\mathrm{b}}$, and Anna Dudek ${ }^{\mathrm{b}}$

a Department of Physical Chemistry, Jagiellonian University, Ingardena 3, 30-060 Kraków, Poland. Fax: +48126340515. E-mail: gzyl@chemia.uj.edu.pl

b Department of Plant Physiology, Polish Academy of Sciences, Podłużna 3, 30-239 Kraków, Poland

* Author for correspondence and reprint requests

Z. Naturforsch. 59c, 60-64 (2004); received April 28/July 7, 2003

The effect of temperatures $\left(15\right.$ and $\left.5{ }^{\circ} \mathrm{C}\right)$ on adsorption parameters of phytohormones at monolayers prepared from a mixture of phospholipids extracted from non-embryogenic (NE) and embryogenic (E) winter wheat calli initiated from inflorescences (inf) and embryos (emb) was studied. The surface parameter values, i.e. limiting area and collapse pressure, were determined using the Langmuir method. Phytohormones 2,4-dichlorophenoxyacetic acid (2,4-D), indole-3-acetic acid (IAA), kinetin, zeatin and zearalenone were investigated. The phytohormones, at a concentration of $0.2 \mu \mathrm{g} / \mathrm{ml}$ dissolved in water, were injected into the subphase. Phospholipids, at the concentration of $2 \mathrm{mg} / \mathrm{ml}$, were spread at the water surface and the monolayer was compressed. The anomalous temperature effect was observed, especially, in non-embryogenic systems. In monolayers obtained from E phospholipids, the temperature effect was dependent on the kind of tissue from which the callus was initiated. Among all the examined phytohormones, the greatest changes (monolayer expansion) were found for IAA and zearalenone. However, this activity depended strongly on the kind of tissue from which the phospholipid mixture was extracted.

Key words: Phytohormones, Phospholipids, Monolayers

\section{Introduction}

Plant hormones (phytohormones) are a group of substances essential for generating and controlling a wide range of responses in plants. Unfortunately, the mechanisms by which phytohormones are recognized and through which the phytohormone signal is transduced into the responses are so poorly understood that more investigations which could help to solve this problem are necessary. Due to the polar character of most of phytohormones, their interaction with phospholipids, main components of plant cell membranes, is possible. Our earlier results (Filek et al., 2002), indicate that phytohormones such as 2,4-D, IAA, kinetin, zeatin and zearalenone can influence the zeta potential of liposomes prepared from phospholipids extracted from plant cells. Additionally, these phytohormones changed the properties of phospholipid monolayers at $25^{\circ} \mathrm{C}$ (Filek et al., 2003). Monolayer properties detected by the Langmuir method provide information about direct interactions in the phase boundary. The proportion between various classes of phospholipids are specific for different cells and could be modi- fied by genetic and environmental conditions. It is well documented that in plants, a change in growth temperature often induces alteration of the fatty acid composition of membrane lipids (Styler et al., 1996). The general trend observed is an increase in the extent of fatty acid unsaturation at lower temperatures. This process is thought to adjust membrane fluidity, and thereby maintain membrane function, over a range of temperatures normally experienced in the natural environment (Styler et al., 1996). It has been suggested that an increase in fatty acid unsaturation tends to maintain the liquid-crystalline phase (Pastori and Trippi, 1995). These physiological changes in lipids composition at lower temperatures could prevent specific lipids/water interaction at the phase boundary. Phospholipid monolayers were prepared as mixtures of polar and hydrophobic parts adequate to the phospholipids extracted from plants.

The aim of the presented experiments was to study the effect of lowering temperature on the influence of plant hormones on the surface properties of monolayers prepared from natural phospholipids extracted from wheat callus cells. Lower 
temperature of callus cultures favours the regeneration process in wheat. The equilibrium between different classes of plant hormones determines the regeneration competence of cells. IAA, 2,4-D, kinetin, zeatin and zearalenone were chosen as plant hormones in order to compare obtained results with earlier ones presented by Filek et al. (2003). Monolayer properties were investigated by Langmuir method at 5 and $15^{\circ} \mathrm{C}$.

\section{Materials and Methods}

Phytohormones were obtained from Sigma Chemical Co. (St. Louis, MO, USA). The following phytohormones were examined: indole-3-acetic acid (IAA), 6-[4-hydroxy-3-methylbut-2-enylamino]purine (zeatin), 2,4-dichlorophenoxyacetic acid (2,4-D), 6-furfurylaminopurine (kinetin), and 6-[10-hydroxy-6-oxo-trans-1-undecyl]-2,4-dihydroxybenzoic acid lactone (zearalenone).

Natural phospholipids (PL) were isolated from winter wheat cells of calli cv. Kamila, as described previously (Filek et al., 2002). Callus cells were initiated from two types of wheat tissue i.e. immature inflorescences (inf) and embryos (emb). Both cultures were in the non-embryogenic (NE) and embryogenic (E) stage. The composition of phospholipid mixtures was determined by thin layer and gas chromatography as described previously (Filek et al., 2003).

The polar part of mixed phospholipids (PPL) contained: phosphatidylcholine dipalmitoyl (PC), phosphatidylethanolamine dipalmitoyl (PE), phosphatidic acid dipalmitoyl (PA), phosphatidyl glicerol dipalmitoyl (PG), phosphatidylserine dipalmitoyl (PS) and phosphatidylinositol dipalmitoyl (PI); the fractions (mol\%) for $\mathrm{NE}$ inf are: $33 \%$ of PC, $31 \%$ PE, $10 \%$ PA, $12 \%$ PG, $8 \%$ PI and $6 \% \mathrm{PS}$; for $\mathrm{E}$ inf : $32 \% \mathrm{PC}, 34 \% \mathrm{PE}, 13 \% \mathrm{PA}$, $10 \% \mathrm{PG}, 6 \% \mathrm{PI}$ and $5 \% \mathrm{PS}$; for NE emb: $33 \%$ PC, $30 \%$ PE, $12 \%$ PA, 14\% PG, $7 \%$ PI and $4 \%$ PS; for E emb 33\% PC, 32\% PE, 13\% PA, 12\% PG, $6 \%$ PI and $4 \%$ PS. The hydrophobic part (HPL) contained (mol\%) for NE inf: $27 \%$ of palmitic acid (16:0), 0.5\% stearic acid (18:0), 7\% oleic acid (18:1), 50.5\% linoleic acid (18:2) and 15\% linolenic acid (18:3); for E inf: 24\% 16:0, 0.5\% 18:0, $2.5 \% 18: 1,54 \% 18: 2$ and $19 \% 18: 3$; for NE emb: $25 \% 16: 0,0.5 \% 18: 0,5.5 \% 18: 1,51 \% \quad 18: 2$ and 18\% 18:3; for $\mathrm{E}$ emb: $29 \%$ 16:0, 0.5\% 18:0, $3 \%$ $18: 1,46.5 \% 18: 2,21 \% 18: 3$. Samples were stored in the dark at $-20^{\circ} \mathrm{C}$.
As the spreading solvent, chloroform/methanol $(4: 1 \mathrm{v} / \mathrm{v})$ mixture was used. Chloroform and methanol were purchased from Sigma Chemical Co. Bidistilled water was used as subphase.

The surface pressure was determined by using a KSV 1000 balance (KSV Instruments Ltd, Helsinki, Finland). The compression was continuous with a rate of $6 \mathrm{~mm} / \mathrm{min}$. The accuracy of measurements was about $\pm 0.01 \mathrm{mN} / \mathrm{m}$ for the surface pressure, $\pm 0.01 \mathrm{~A}^{2} /$ molecule for the area, and $\pm 0.05^{\circ} \mathrm{C}$ for the temperature as controlled by a U3 thermostat. The measurements were performed at $15^{\circ} \mathrm{C}$. Before each experiment, the surface was cleaned until the value of surface pressure remained about zero on reducing the surface area. The phytohormones, at a concentration of $0.2 \mu \mathrm{g} / \mathrm{ml}$ dissolved in water, were injected into subphase by using a Hamilton microlitre syringe. Then, $35 \mu \mathrm{l}$ of phospholipids $(2 \mathrm{mg} / \mathrm{ml})$ were spread at the water surface and the monolayer was compressed.

\section{Results and Discussion}

Table I shows the surface parameters obtained from the surface pressure $(\pi)$ - mean molecular area $(A)$ isotherms recorded for monolayers formed by phospholipids extracted from embryogenic (E) and non-embryogenic (NE) wheat calli initiated from embryos (emb) and inflorescences (inf), on water solutions of phytohormones, at 5 and $15^{\circ} \mathrm{C}$. Values of limiting area and collapse pressure were determined as the surface parameters. The limiting area per molecule $\left(A_{\text {lim }}\right)$ is the area per molecule under maximum packing conditions extrapolated to zero surface pressure (Clint, 1992). Collapse pressure $\left(\pi_{\mathrm{coll}}\right)$ is the maximum pressure to which a monolayer can be compressed without detectable expulsion of molecules from the Langmuir film (Gaines, 1966; Birdi, 1989).

In our experiments, low phytohormone concentrations were applied because these compounds exist in plants in very small amounts having, however, a substantial influence on the physiological activity of plants. Phytohormones were not surface-active under experimental conditions. However, their presence in the subphase influenced the surface parameters of phospholipid monolayers and caused their expansion. Therefore, it is suggested that phospholipids stimulated the adsorption of phytohormones dissolved in the subphase. Among all the examined phytohormones, the 
Table I. The limiting area per molecule $\left(A_{\lim }\right)$ and collapse pressure $\left(\pi_{\text {coll }}\right)$ for monolayers of phospholipids extracted from non-embryogenic (NE) and embryogenic (E) inflorescences (inf) and embryos (emb), in presence of phytohormones (zeatin, kinetin, IAA, 2,4-D, zearalenone) in water subphase, at 5 and $15^{\circ} \mathrm{C}$. The average value for 3 independent experiments $\pm \mathrm{SD}$ is given.

\begin{tabular}{|c|c|c|c|c|c|}
\hline \multicolumn{2}{|c|}{ Treatment } & \multicolumn{2}{|c|}{$A_{\lim }\left[\mathrm{A}^{2} /\right.$ molecule $]$} & \multicolumn{2}{|c|}{$\pi_{\text {coll }}[\mathrm{mN} / \mathrm{m}]$} \\
\hline Phytohormone & Object & $5^{\circ} \mathrm{C}$ & $15^{\circ} \mathrm{C}$ & $5^{\circ} \mathrm{C}$ & $15^{\circ} \mathrm{C}$ \\
\hline \multirow[t]{4}{*}{ Zeatin } & NE inf & $50.28 \pm 0.05$ & $64.00 \pm 0.07$ & $42.24 \pm 0.03$ & $35.01 \pm 0.04$ \\
\hline & $\mathrm{E}$ inf & $69.08 \pm 0.04$ & $67.21 \pm 0.06$ & $40.59 \pm 0.03$ & $33.86 \pm 0.03$ \\
\hline & $\mathrm{NE}$ emb & $58.61 \pm 0.04$ & $70.74 \pm 0.04$ & $40.83 \pm 0.04$ & $36.09 \pm 0.03$ \\
\hline & $\mathrm{E}$ emb & $61.31 \pm 0.07$ & $74.19 \pm 0.06$ & $38.39 \pm 0.03$ & $34.37 \pm 0.04$ \\
\hline \multirow{4}{*}{ Kinetin } & $\mathrm{NE}$ inf & $51.24 \pm 0.04$ & $63.88 \pm 0.04$ & $41.54 \pm 0.03$ & $35.97 \pm 0.03$ \\
\hline & $E$ inf & $68.09 \pm 0.03$ & $67.37 \pm 0.05$ & $41.06 \pm 0.04$ & $34.27 \pm 0.03$ \\
\hline & NE emb & $59.39 \pm 0.05$ & $71.53 \pm 0.04$ & $41.87 \pm 0.03$ & $37.10 \pm 0.05$ \\
\hline & $\mathrm{E}$ emb & $62.48 \pm 0.09$ & $74.79 \pm 0.03$ & $40.29 \pm 0.04$ & $36.07 \pm 0.05$ \\
\hline \multirow[t]{4}{*}{ IAA } & $\mathrm{NE}$ inf & $56.82 \pm 0.05$ & $65.35 \pm 0.06$ & $41.69 \pm 0.05$ & $34.59 \pm 0.03$ \\
\hline & $E$ inf & $74.85 \pm 0.03$ & $70.42 \pm 0.04$ & $41.50 \pm 0.04$ & $36.96 \pm 0.04$ \\
\hline & $\mathrm{NE}$ emb & $62.51 \pm 0.04$ & $70.50 \pm 0.07$ & $41.98 \pm 0.03$ & $39.71 \pm 0.03$ \\
\hline & $\mathrm{E} \mathrm{emb}$ & $66.79 \pm 0.06$ & $77.94 \pm 0.03$ & $39.86 \pm 0.04$ & $36.02 \pm 0.03$ \\
\hline \multirow[t]{4}{*}{$2,4-\mathrm{D}$} & $\mathrm{NE}$ inf & $50.66 \pm 0.05$ & $62.04 \pm 0.03$ & $41.10 \pm 0.04$ & $37.10 \pm 0.05$ \\
\hline & $E \inf$ & $68.76 \pm 0.06$ & $66.01 \pm 0.05$ & $41.35 \pm 0.03$ & $35.44 \pm 0.03$ \\
\hline & $\mathrm{NE}$ emb & $58.85 \pm 0.06$ & $70.80 \pm 0.03$ & $41.94 \pm 0.03$ & $40.42 \pm 0.04$ \\
\hline & $\mathrm{E}$ emb & $60.77 \pm 0.06$ & $74.55 \pm 0.05$ & $39.52 \pm 0.03$ & $33.26 \pm 0.04$ \\
\hline \multirow[t]{4}{*}{ Zearalenone } & $\mathrm{NE}$ inf & $54.88 \pm 0.04$ & $64.37 \pm 0.06$ & $41.89 \pm 0.04$ & $36.22 \pm 0.03$ \\
\hline & $E$ inf & $68.02 \pm 0.05$ & $68.46 \pm 0.07$ & $41.08 \pm 0.05$ & $33.60 \pm 0.03$ \\
\hline & $\mathrm{NE}$ emb & $60.01 \pm 0.06$ & $70.47 \pm 0.05$ & $41.28 \pm 0.05$ & $37.94 \pm 0.04$ \\
\hline & $\mathrm{E}$ emb & $62.94 \pm 0.09$ & $74.12 \pm 0.05$ & $39.90 \pm 0.03$ & $35.09 \pm 0.03$ \\
\hline
\end{tabular}

greatest expansion of phospholipid monolayers (the highest values of the limiting areas) was observed when IAA was injected into the subphase. The limiting area values obtained for phospholipid monolayers with IAA were about $1-6.5 \mathrm{~A}^{2} /$ molecule higher than in the presence of other phytohormones. The greatest differences in activity between IAA and other phytohormones were observed for embryogenic systems (E). In the case of non-embryogenic systems (NE), a greater influence of IAA was observed at the lower temperature $\left(5^{\circ} \mathrm{C}\right)$. Its synthetic equivalent $2,4-\mathrm{D}$ influenced phospholipid monolayers to a lesser extent - the $A_{\text {lim }}$ values were much lower and comparable with those recorded for cytokinins. As far as cytokinins are concerned, both zeatin and its synthetic equivalent, kinetin, expanded monolayers to an equal degree. The only differences were noticed for phospholipid monolayers prepared from calli initiated from embryos (emb), where a little higher activity of the synthetic phytohormone was observed $\left(A_{\lim }\right.$ values for kinetin were about $0.6-$ $1.1 \mathrm{~A}^{2} /$ molecule higher than for zeatin).

Zearalenone, investigated as a phytohormoneactive substance in embryogenic process (in preparation), showed relatively high activity on phos- pholipid monolayers, however, it was lower than observed for IAA. A comparison of $A_{\text {lim }}$ values obtained for monolayers of phospholipids extracted from inf and emb leads to the conclusion that zearalenone was particularly active in emb systems at the lower temperature $\left(5^{\circ} \mathrm{C}\right)$ and in inf systems at the higher temperature $\left(15^{\circ} \mathrm{C}\right)$ independently of the embryogenic state. The only exception was NE inf monolayer for which great expansion was observed regardless of the temperature. NE inf system contains more saturated chains than the other PL systems. Since zearalenone is the most hydrophobic among the examined phytohormones, its greatest influence on NE inf monolayer can be related to its interactions with the hydrophobic part of phospholipids.

Higher values of $A_{\text {lim }}$ found for E (inf and emb) than NE (inf and emb) phospholipid monolayers suggesting lower molecular packing of embryogenic membranes are consistent with our earlier results (Filek et al., 2003). The E tissue was characterised by a higher content of unsaturated fatty acid 18:3. The presence of three double bonds caused a bend in the chain and its tilting towards the surface. As a consequence, the distance between chains increased and then the van der Waals 
interactions decreased. Therefore, embryogenic systems were characterized by a less coherent structure. This was also the reason for lower stability against the collapse process observed for these monolayers, as was indicated by lower $\pi_{\text {coll }}$ values at both temperatures.

The temperature has a great influence on the values of the limiting area $A_{\mathrm{lim}}$. Higher values of surface areas were observed at 15 than $5^{\circ} \mathrm{C}$ (exept $\mathrm{E}$ inf, where changes of temperature insignificantly influenced $A_{\text {lim }}$ ). Usually, when the temperature increases, the limiting area also increases as a consequence of higher mobility assumed by the hydrocarbon chains. Such an effect was observed here when the temperature rose from 5 to $15^{\circ} \mathrm{C}$. A comparison of these results with earlier experiments (Filek et al., 2003) let us to the conclusion that a further increase in the temperature by 10 degrees caused a decrease in the limiting area for all examined monolayers (Fig. 1). This phenomenon has been reported (Caminati et al., 1991) and is called an anomalous temperature effect. It is ascribed to the dehydratation process of polar head groups of phospholipids. With the temperature increase, hydrogen bonds between polar groups of phospholipids and between them and water molecules break down, some water molecules are transferred from the coordination shell of polar groups to the bulk phase. This causes the rearrangement of the molecules at the interface, the distance between hydrophilic parts of lipids decreases, the electrostatic forces acting between polar heads

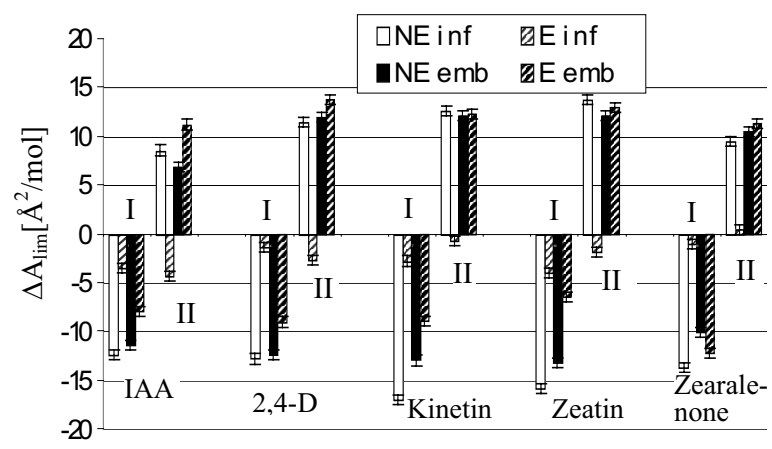

Fig. 1. The influence of phytohormones IAA, 2,4-D, kinetin, zeatin and zearalenone on relative changes of limited area $\left(A_{\text {lim }}\right)$ calculated as a difference between values obtained at 5 and $15^{\circ} \mathrm{C}$ (I) as well as 15 and $25^{\circ} \mathrm{C}$ (II). Monolayers were formed from phospholipids of non- (NE) and embryogenic (E) winter wheat cultures initiated from inflorescences (inf) and embryos (emb). Each value is a mean \pm SD for three experiments. and van der Waals interactions of hydrocarbon chains become stronger. If one looks at the Fig. 1, it is easy to see that the anomalous temperature effect was much greater for non-embryogenic than for embryogenic tissue (the greater contraction of the limiting area for NE). This can be explained by the composition of phospholipid mixture. Nonembryogenic tissue contains a higher percentage of phosphatidylinositol which possesses the cyclic structure with five hydroxylic groups able to form hydrogen bonds with themselves and with water molecules.

Phytohormones present at phospholipid surface maintained the observed anomalous temperature effect. Moreover, this effect was observed even in the lower temperature range $\left(5-15^{\circ} \mathrm{C}\right)$ for $\mathrm{E}$ inf monolayer in the presence of phytohormones in the subphase (except of zearalenone) and is stronger for auxins (IAA and 2,4-D) than for cytokinins (zeatin and kinetin). However, the area contraction found for $\mathrm{E}$ inf monolayer is small in comparison with the values of molecular area expansion observed for all other systems. In the other systems (i.e. NE inf, E emb and NE emb) the normal temperature effect was observed. For a given phytohormone the area expansion was similar independently of the kind of explant used for phospholipid extraction (Fig. 1), although in the case of auxin, a little greater expansion of $E$ emb monolayers was noticed when the temperature grew from 5 to $15^{\circ} \mathrm{C}$.

In the higher temperature range $\left(15-25^{\circ} \mathrm{C}\right)$, all examined systems showed the anomalous temperature effect. It is interesting to notice that in this case the lowest $\Delta A_{\text {lim }}$ values (i.e. $A_{\text {lim }}$ at $25^{\circ} \mathrm{C}-$ $A_{\text {lim }}$ at $15^{\circ} \mathrm{C}$ ) were also obtained for E inf monolayers, what was probably caused by the fact that the area contraction (and therefore the loss of water molecules from the surface layer) had already started at $15^{\circ} \mathrm{C}$. However, the stronger an anomalous temperature effect was observed for cytokinins than for auxins contrary to the results obtained in the lower temperature range where the stronger an anomalous temperature effect was observed for auxins (the greatest area contraction). Generally, greater changes in molecular area were observed for the temperature increase from $15^{\circ} \mathrm{C}$ to $25^{\circ} \mathrm{C}$ than from $5^{\circ} \mathrm{C}$ to $15^{\circ} \mathrm{C}$. In the first case (higher temperature range), the area contraction was connected with the dehydratation process, therefore the water molecules were transferred from the surface to the bulk, whereas in the latter 
(lower temperature range) extension was related only to the greater mobility assumed by hydrocarbon chains as the temperature was growing.

Collapse pressure values were also affected by the temperature - when the temperature rose from 5 to $15^{\circ} \mathrm{C}, \pi_{\text {coll }}$ decreased by several units (Fig. 2). However, if the temperature increased further up to $25^{\circ} \mathrm{C}$, an increase in collapse pressures was noticed. Therefore, the lowest stability of monolayer against the collapse process was observed at $15^{\circ} \mathrm{C}$. The kind of phytohormone had a great influence on the change in the monolayer stability caused by the change of temperature. In the case of IAA, the highest $\Delta \pi_{\text {coll }}$ (the differences between $\pi_{\text {coll }}$ values at upper and lower temperature, i.e. $\pi_{\text {coll }}$ at $25^{\circ} \mathrm{C}-\pi_{\text {coll }}$ at $15^{\circ} \mathrm{C}$ and $\pi_{\text {coll }}$ at $15^{\circ} \mathrm{C}-\pi_{\text {coll }}$ at $5^{\circ} \mathrm{C}$ ) values were observed for $\mathrm{NE}$ inf monolayers in both temperature ranges. For $2,4-\mathrm{D}$, the highest $\Delta \pi_{\text {coll }}$ values were found in embryogenic systems. Zearalenone showed an interesting behaviour in $\mathrm{E}$ inf system: in the lower temperature range $\left(5-15^{\circ} \mathrm{C}\right)$, the highest $\Delta \pi_{\text {coll }}$ value was observed for this system, whereas in the upper temperature range the lowest one. As it was mentioned earlier, the temperature growth till $25^{\circ} \mathrm{C}$

Birdi K. S. (1989), Experimental methods and procedures in monolayers. In: Lipid and Biopolymer Monolayers at Liquid Interfaces. Plenum Press, New York, pp. 27-56.

Caminati G., Senatra D., and Gabrielli G. (1991), 1-Hexanol and 1-tetradecanol adsorption at the water-oil interface. Langmuir 7, 1969-1974.

Clint J. H. (1992), Insoluble monolayers. In: Surfactant Aggregation. Blackie \& Son Ltd, Glasgow, pp. 33-58.

Filek M., Zembala M., and Szechyñska-Hebda M. (2002), The influence of phytohormones on zeta potential and electrokinetic charges of winter wheat cells. Z. Naturforsch. 57c, 696-704.

Filek M., Gzyl B., and Dudek A. (2003), The influence of phytohormones on properties of wheat phospholipid monolayers at water-air interface. Cell. Mol. Biol. Lett. (in press).

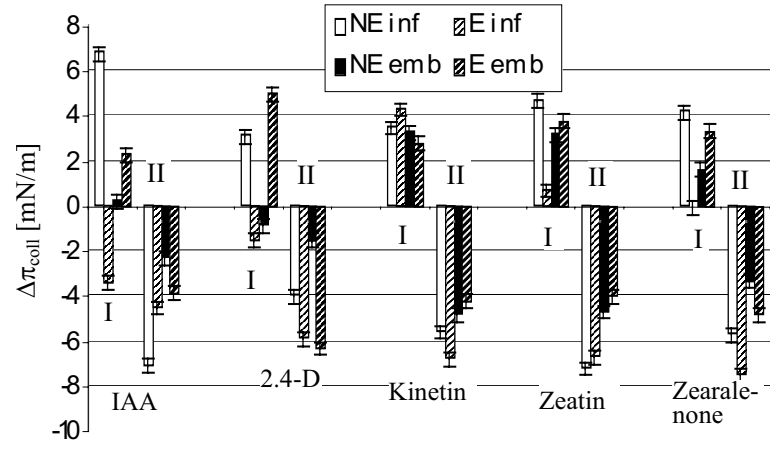

Fig. 2. The influence of phytohormones IAA, 2,4-D, kinetin, zeatin and zearalenone on relative changes of collapsed pressure $\left(\pi_{\text {coll }}\right)$ calculated as a difference between values obtained at 5 and $15^{\circ} \mathrm{C}$ (I) as well as 15 and $25^{\circ} \mathrm{C}$ (II). Monolayers were formed from phospholipids of non- (NE) and embryogenic (E) winter wheat cultures initiated from inflorescences (inf) and embryos (emb). Each value is a mean \pm SD for three experiments.

causes an increase in the monolayer stability against the collapse process. However, in the case of auxins, the opposite effect was observed in $\mathrm{E}$ inf systems - negative $\Delta \pi_{\text {coll }}$ values were found.

Gaines L. G. Jr. (1966), The stability of spread films. In: Insoluble Monolayers at Liquid-Gas Interfaces. John Wiley \& Sons, Inc., New York, pp. 143-156.

Klyachko G. L., Tsogli L. N., Doucha J., Kopetskii J., Shebalina I. B., and Semenko V. E. (1999), Desaturation of fatty acids as an adaptive response to shifts in light intensity. Physiol. Plant. 107, 240-249.

Los D. A. (1997), Fatty acids and desaturases: Adaptive expression and principles of regulation. Russ. J. Plant Physiol. 44, 458-469.

Pastori G. M. and Trippi V. S. (1995), Fatty acid composition in water- and oxygen-stressed leaves of maize and wheat strains. Phytochemistry 40, 45-48.

Styler E. H., Whitaker B. D., Kitto S. L., and Hoover D. G. (1996), Lipid composition of microsomes from heat-stressed cell suspension cultures. Phytochemistry 41, 187-191. 